\title{
A centrosomal view of CNS growth
}

\author{
Murielle Saade*, Jose Blanco-Ameijeiras, Elena Gonzalez-Gobartt and Elisa Martí
}

\begin{abstract}
Embryonic development of the central nervous system (CNS) requires the proliferation of neural progenitor cells to be tightly regulated, allowing the formation of an organ with the right size and shape. This includes regulation of both the spatial distribution of mitosis and the mode of cell division. The centrosome, which is the main microtubule-organizing centre of animal cells, contributes to both of these processes. Here, we discuss the impact that centrosome-mediated control of cell division has on the shape of the overall growing CNS. We also review the intrinsic properties of the centrosome, both in terms of its molecular composition and its signalling capabilities, and discuss the fascinating notion that intrinsic centrosomal asymmetries in dividing neural progenitor cells are instructive for neurogenesis. Finally, we discuss the genetic links between centrosome dysfunction during development and the aetiology of microcephaly.
\end{abstract}

KEY WORDS: Organ growth, CNS, Interkinetic nuclear migration, Asymmetric cell division, Centrosome, Growth factors, Primary microcephaly

\section{Introduction}

During embryonic development in higher vertebrates, the brain and anterior spinal cord are formed through primary neurulation of the embryonic neural plate, which produces a hollow neural tube (NT) that acts as the primordium of the central nervous system (CNS) (Greene and Copp, 2014). Along its entire anterior-to-posterior axis, the lumen of the NT is covered by a single type of neuroepithelial cell, termed a primary neural progenitor cell (NPC), from which all neural cell types will be generated. NPCs are specified in discrete domains with distinct transcriptional states in response to the activity of secreted proteins (Addison and Wilkinson, 2016; Cohen et al., 2013; Gupta and Sen, 2016; Le Dréau and Martí, 2012; Sousa and Fishell, 2010; Ulloa and Marti, 2010).

During development, primary NPCs proliferate in a tightly controlled manner, exhibiting distinct growth rates along the axis of the NT. The different rates of growth in the anterior and posterior NT are reflected in the enlargement of the brain chambers, which give rise to the primary anatomical structures in the brain. The main divisions initially formed in the anterior part of the CNS are the forebrain (prosencephalon), midbrain (mesencephalon) and hindbrain (rhombencephalon); these are followed caudally by the spinal cord (Fig. 1A). The forebrain comprises two telencephalic vesicles, the dorsal half of which is specified as the primordium of the cerebral cortex (Fig. 1B). At early developmental stages, key features that are important for NPC expansion are conserved

\footnotetext{
Department of Developmental Biology, Instituto de Biología Molecular de Barcelona, Parc Científic de Barcelona, Baldiri i Reixac 20, Barcelona 08028, Spain.

*Author for correspondence (msabmc@ibmb.csic.es)

along the CNS, including within the cerebral cortex and the spinal cord (Fig. 1B-D). However, later in development, NPCs in the developing cerebral cortex are organized into two germinal layers the ventricular zone (VZ) and the subventricular zone (SVZ) - and are subject to increasing layers of complexity. These features of cortical NPCs and neurogenesis have been the subject of excellent recent reviews (Florio et al., 2017; Heide et al., 2017; Johnson and Walsh, 2017; Wilsch-Bräuninger et al., 2016) and will not be discussed further here.

Here, we aim to highlight features of primary NPCs that regulate the early growth of the embryonic CNS, de-regulation of which can cause neurodevelopmental disorders such as primary microcephaly. In particular, we discuss mechanisms involving the centrosome the main microtubule-organizing centre (MTOC) in animal cells. We highlight how the centrosome impacts the process of interkinetic nuclear migration, which not only serves to expose dividing NPCs to the signalling-rich NT lumen environment, but also affects the shaping of the overall growing CNS. We also discuss the intrinsic properties of the centrosome and the emerging notion that centrosome asymmetries can instruct the outcome of NPC division. Moreover, based on recent findings, we highlight how growth factors, known to play a role in the generation of cell diversity during CNS development, also contribute to centrosome maturation and signalling, and thus regulate the mode of NPC division. Finally, given that many of the causative mutations for primary microcephaly affect genes encoding centrosome-related proteins (Gilmore and Walsh, 2013; Jayaraman et al., 2018), we briefly discuss how studies of the centrosome represent an interesting research direction for improving our understanding of neurodevelopmental disorders such as microcephaly.

\section{Centrosome-dependent interkinetic nuclear migration confines mitosis to the apical area}

The NPCs that form the embryonic primordium of the CNS are organized as a pseudostratified epithelium in which elongated cells contact both the apical and basal laminae, with their nuclei adopting distinct positions along the apicobasal cell axis (Fig. 2A). During the G1 phase of the cell cycle, the nuclei of NPCs born at the apical surface of the neuroepithelium move toward the basal side. After completing $\mathrm{S}$ phase contacting the basal portion of the neuroepithelium, the nuclei return to the apical surface, where they undergo mitoses as their parent cells did. Collectively, these processes are referred to as interkinetic nuclear migration (INM, Fig. 2A) (Langman et al., 1966; Sauer, 1935).

In NPCs, the centrosome is anchored at the apical surface of the cell (Fig. 2B), serving as the base for the primary cilium (Dubreuil et al., 2007; Goetz and Anderson, 2010). Experimental observations indicate that the centrosome behaves as an anchor point for an apical-ward force that pulls the nucleus during G2 phase of the cell cycle. As such, the forces that drive apical nuclear migration within the $\mathrm{VZ}$ require the activity of centrosomal proteins, such as SAS-4 (CENPJ in mammals), Cep120, TACCs and Hook3 (Ge et al., 2010; Insolera et al., 2014; Xie et al., 2007). In parallel, and in line with the 
A

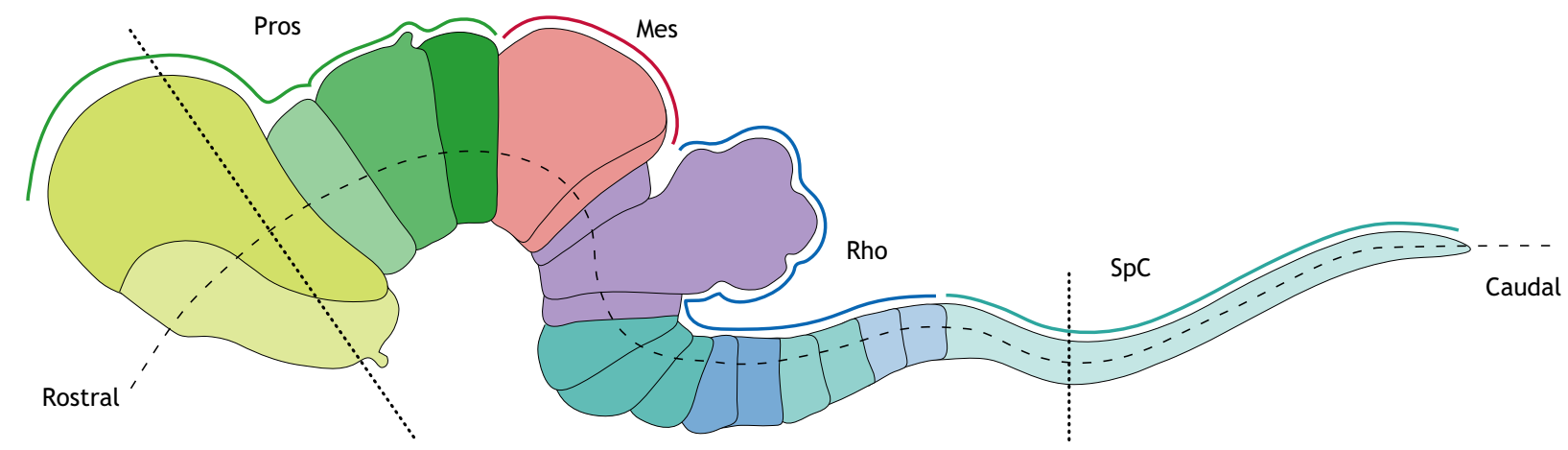

B

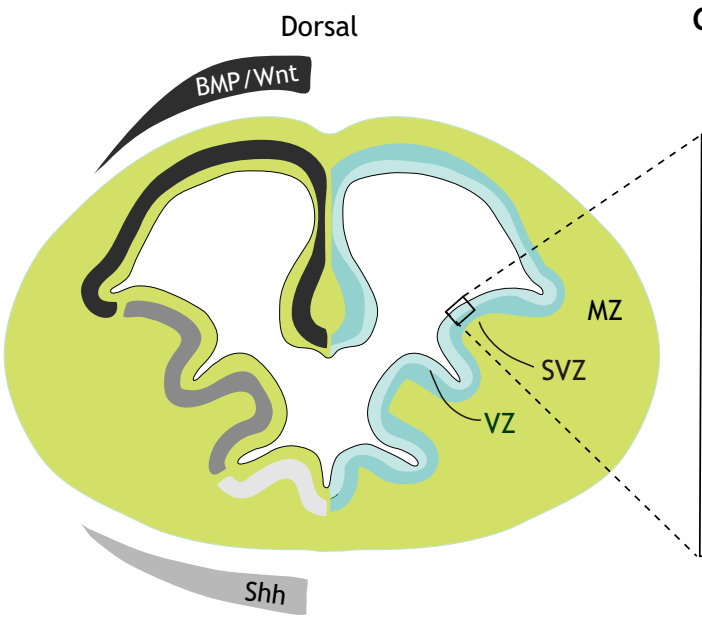

Ventral
C

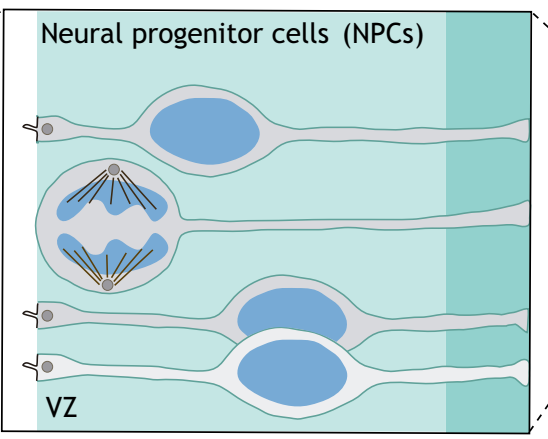

C D

Dorsal

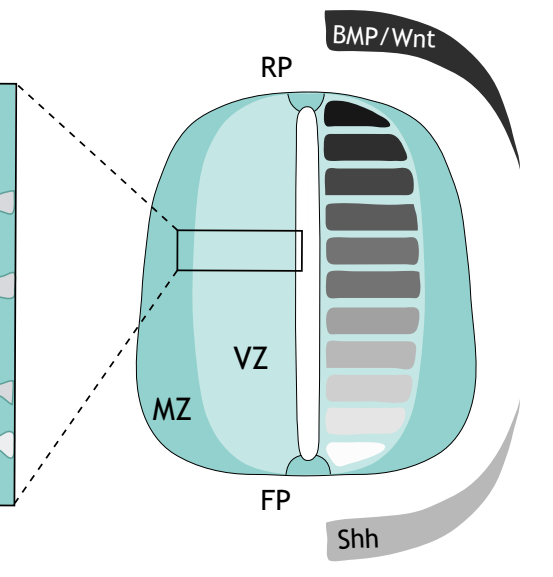

Ventral

Fig. 1. The embryonic central nervous system. (A) Diagram of a vertebrate embryo (mouse $\sim$ E8-10) central nervous system, showing rostral to caudal regionalization into the forebrain (prosencephalon, Pros), midbrain (mesencephalon, Mes), hindbrain (rhombencephalon, Rho), and the caudal spinal cord $(\mathrm{SpC})$. (B) Diagram of a transverse section through the telencephalon. The main telencephalic subdivisions along the dorsal ventral axis are generated in

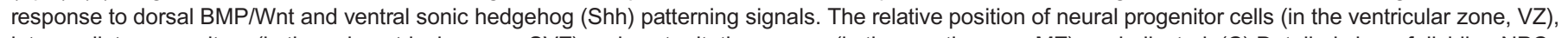

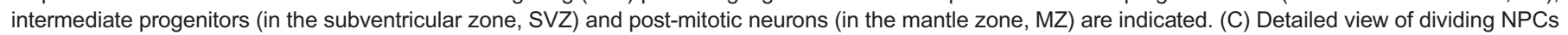
that occupy the VZ lining the entire neural tube lumen. NPCs are present as elongated cells that contact both the apical and basal laminae, with their nuclei

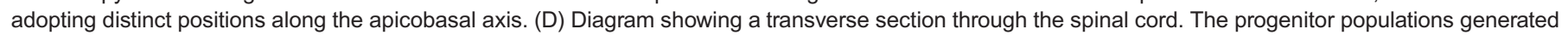

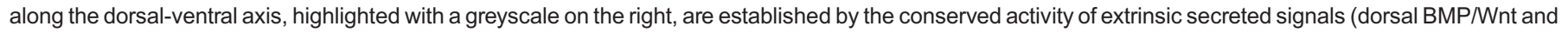
ventral Shh). The relative position of NPCs (in the ventricular zone, VZ) and post-mitotic neurons (in the mantle zone, MZ) is shown. FP, floor plate; RP, roof plate.

role of the centrosome as a MTOC and the observation that intact microtubules are required for INM (Kosodo et al., 2011; Lee and Norden, 2013; Reinsch and Gonczy, 1998), microtubules and their associated motor proteins also contribute to the molecular machinery of INM (Tsai et al., 2010). Indeed, mutations in genes encoding dynein-interacting proteins, such as lissencephaly-1 (Lis1; also known as Pafah1b1), dynactin 1 and laminin $\gamma 1$ (Lamc1), give rise to perturbed apical-ward nucleokinesis and, consequently, mitoses throughout the neuroepithelium (Del Bene et al., 2008; Feng et al., 2000; Tanaka et al., 2004; Tsuda et al., 2010). Interestingly, in shorter NPCs, such as those in the zebrafish developing retina, there must be some centrosome-independent mechanism of INM, as it appears that once apical INM is triggered a 'point of no return' is passed so that apical mitoses take place independently of centrosome position (Strzyz et al., 2015). Whether this mechanism is conserved in other neuroepithelia is not known.

The connection between the microtubule network controlling INM and the nuclear envelope is mediated by KASH-domain proteins (Syne proteins; also known as nesprin proteins), which form a complex with SUN-domain proteins in the nuclear envelope (Fig. 2B). Following the hypothesis of the centrosome as an anchor point for apical-ward INM, this microtubule network-nuclear envelope connection must be kept intact to allow such a nuclear migration. Indeed, experiments in knockout mice have revealed that the SUNdomain proteins SUN1 and SUN2 and the KASH domain proteins Syne1 and Syne2 are required for the apical migration of nuclei along microtubules toward the apical centrosome (Ge et al., 2010; Schenk et al., 2009; Xie et al., 2007; Zhang et al., 2009) (Fig. 2B).

The rationale behind the striking arrangement and dynamics of NPCs has classically been explained as a mechanism to pack more NPCs into a limited space. As such, INM serves to vary the distances of nuclei from the apical and basal surfaces, thereby allowing more NPCs to remain associated with the limited apical/ basal surfaces than would be possible in a columnar epithelium. However, according to the original description of INM, "the mitoses are confined to the region of the lumen not only because nuclei of that region divide, but because a nucleus that is about to divide moves to the region of the lumen to do so' (Sauer, 1935), indicating that it might be beneficial to send the nucleus to the apical area prior to entering mitosis. One possible advantage of sending the nucleus to the apical area during the G2 phase of the cell cycle is that it makes the centrosome available for entry into division. Supporting 


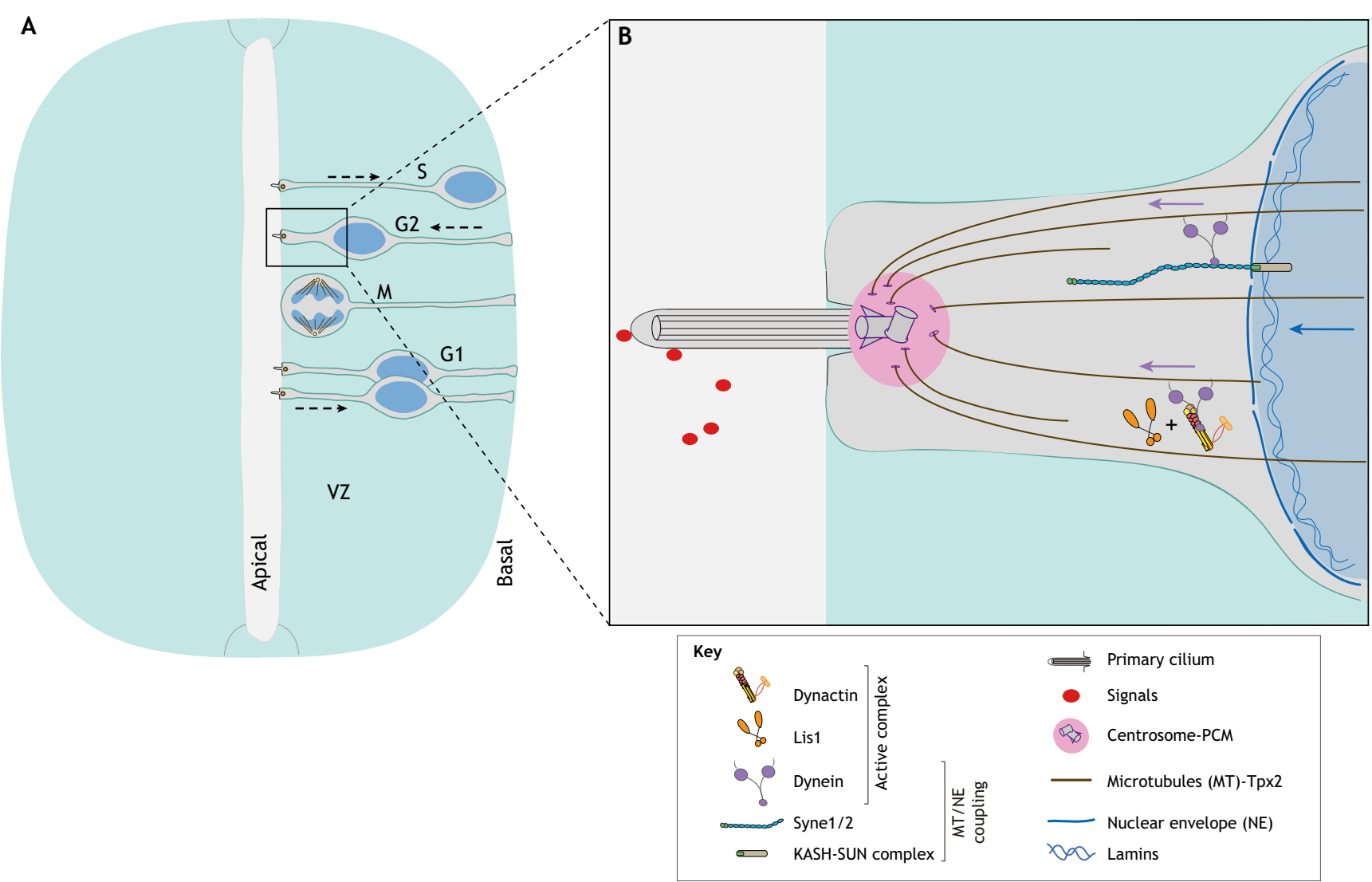

Fig. 2. Interkinetic nuclear migration in NPCs. (A) Diagram of a transverse section through the spinal cord. The nuclei of dividing NPCs occupy different apicobasal positions depending on the phase of the cell cycle (S, G2, M, G1). Dashed arrows indicate the direction of nuclear migration. (B) Diagram of the apical pole of an NPC in the G2 phase of the cell cycle. The primary cilium points to the NT lumen. The centrosome at the cilium base organizes microtubules to pull the nucleus apicalwards (blue arrow). A dynein complex linked to the nuclear envelope activates nuclear apical migration (purple arrows).

this idea, mitosis is in part triggered by a cascade of proteins localized to the centrosome, culminating in the activation of aurora kinase A and, subsequently, in the activation of the cyclin $\mathrm{B} / \mathrm{Cdk} 1$ complex (Hirota et al., 2003; Jackman et al., 2003), which may function as a checkpoint for centrosome availability for division. The microtubule-dependent apical-ward transition of the nucleus in G2 phase might also facilitate the equal inheritance of apical attachments, thereby ensuring cohesion of the tissue despite a high proliferation rate. Additionally, increasing the exposure of NPCs to signalling pathways and molecules that function at the apical surface, such as Notch, which is known to be required for maintaining the progenitor character of NPCs (Hatakeyama et al., 2014; Ohata et al., 2011), might also be among the benefits of apical mitoses. Hence, besides affecting cell packing, INM could restrict the location of mitosis to particular regions of the NT lumen, thereby impacting the signals received by NPCs. These signals might be instructive for the outcome of cell division, as discussed below, and thus are important for CNS growth.

\section{Intrinsic centrosomal asymmetries in dividing neural progenitor cells are instructive for neurogenesis}

Embryonic CNS growth requires a finely tuned balance between the different modes of divisions that NPCs undergo: symmetric proliferative divisions ensure expansion of the progenitor pool by generating two daughter cells with identical progenitor potential, whereas asymmetric divisions generate one daughter cell with progenitor potential and one daughter cell with a more restricted potential, which is then committed to neuronal differentiation
(Fig. 3). However, cell division in general is intrinsically asymmetric as a consequence of differences in the centrosomes that are passed on to the daughter cells (Fig. 3). Before entering mitosis, the centrosome replicates in a semi-conservative manner, forming one centrosome that retains the mother centriole and another that receives the daughter centriole. As we discuss below, this centrosome asymmetry, which relates to centrosome age, structure, molecular composition, MTOC capabilities, and the recruitment of signalling components, can influence the fate of NPC divisions and, hence, the expansion of the progenitor pool.

NPCs inherit one centrosome consisting of a pair of centrioles surrounded by amorphous pericentriolar material (PCM). The two centrioles differ in their structure and function. The older 'mother' centriole possesses distinct sets of projections at its distal ends called subdistal and distal appendages, which bear specific proteins such as CEP164, CEP170, cenexin (also known as Odf2) and ninein that are implicated in the anchoring of microtubules, cilia formation and docking of the basal body at the plasma membrane (Graser et al., 2007; Ishikawa et al., 2005; Ou et al., 2002; Schmidt et al., 2012; Welburn and Cheeseman, 2012). In contrast to the mother centriole, the younger 'daughter' centriole lacks these appendages. Full acquisition of appendages by the daughter centriole is not achieved until at least one and a half cell cycles later (Hoyer-Fender, 2010; Mahen and Venkitaraman, 2012). Importantly for CNS growth, this built-in centrosome asymmetry has an impact on the fate of the daughter cells. Both in the developing mouse cortex (Paridaen et al., 2013; Wang et al., 2009) and in the chick spinal cord (Saade et al., 2017; Tozer et al., 2017) the centrosome retaining 

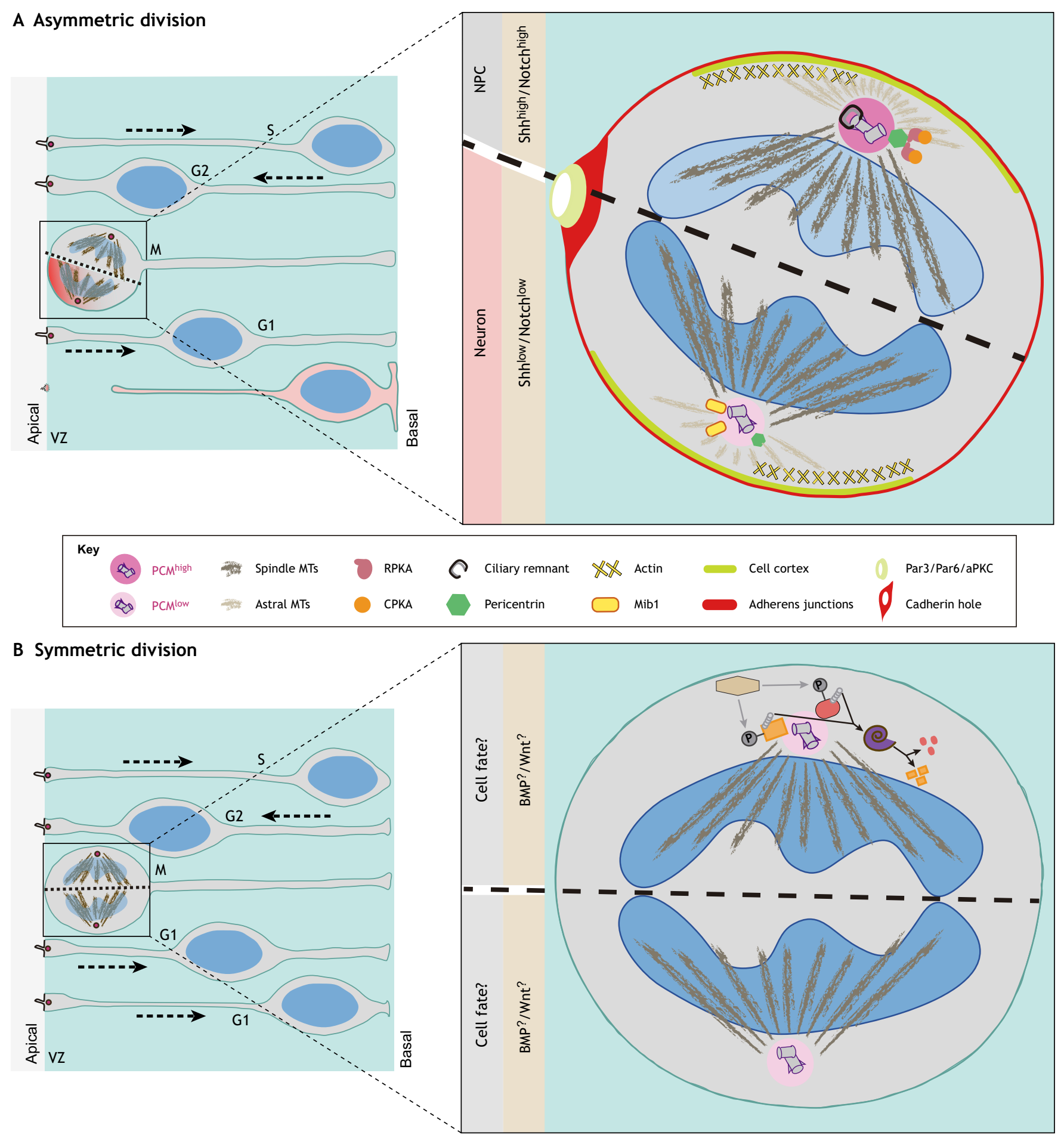

\begin{tabular}{|c|c|c|c|c|c|c|}
\hline \multicolumn{7}{|l|}{ Key } \\
\hline \multirow{2}{*}{$\Leftrightarrow$ Centrosome } & Smad1 & (P) & Phosphorylation & GSK3 & pep & Degraded Smad1 \\
\hline & $\beta$-Catenin & $\mathrm{CeO}$ & Ubiquitylation & Proteasome & $\therefore$ & Degraded $\beta$-catenin \\
\hline
\end{tabular}

Fig. 3. NPC modes of cell division. (A,B) An asymmetrically dividing NPC generates one NPC and one differentiating neuron (pink, A), whereas a symmetrically dividing NPC generates two NPCs (B). (A) An asymmetrically dividing NPC shows asymmetric recruitment of PKA and Mib1 and asymmetric astral microtubule (MT) nucleation. Association of the astral MTs with the cell cortex defines the positioning of the mitotic spindle relative to the axis of polarity, favouring asymmetric inheritance of cell fate components. CPKA, protein kinase A catalytic subunit; RPKA, protein kinase A regulatory subunit; Mib1, Mind bomb-1. (B) A symmetrically dividing NPC shows centrosomal recruitment of Smad1 and $\beta$-catenin and the ubiquitin proteasome system. The final impact of this distribution of BMP and Wnt signalling components on the division mode of NPCs and the cell fate of daughter cells is still not well described. Grey arrows indicate phosphorylation; black arrows indicate proteasome-dependant degradation. 
the old mother centriole is preferentially inherited by the NPC, whereas the centrosome containing the daughter (new mother) centriole is inherited by the delaminating and differentiating neuron, which leaves the VZ.

This centrosomal asymmetry has a number of downstream effects on NPC-derived cells. For instance, maturation of the daughter centriole is required for correct NPC function. The process of centrosome maturation is characterized by drastic expansion of the pericentriolar material and a robust increase in MTOC activity. During this event, proteins such as centrin and ninein are delivered to the centrosome along microtubules via a dynein/dynactin-dependent process (Dammermann and Merdes, 2002). Accordingly, the removal of mature centriole-specific proteins, including ninein (Wang et al., 2009), WDR62 and ASPM (Gai et al., 2016; Jayaraman et al., 2016), is sufficient to cause premature depletion of progenitor cells from the VZ and to impair CNS growth. Other proteins are recruited to the centrosome in a microtubule-independent manner by interacting with scaffold proteins, such as AKAP9 and pericentrin (Gillingham and Munro, 2000), which contain a localization domain (PACT domain) that targets the centrosome and serves to recruit structural and regulatory components such as $\gamma$-tubulin, microtubule binding proteins and signalling enzymes involved in microtubule nucleation (Almada et al., 2017). Supporting the relevance of centrosomal scaffold proteins in the control of the mode of NPC division, the removal of pericentrin triggers neurogenic divisions both in the chick spinal cord (Saade et al., 2017) and in the developing mouse cortex (Buchman et al., 2010). Surprisingly, pericentrin expression, together with other genes involved in centrosome maturation, appears to be regulated by the sonic hedgehog (Shh)/Gli signalling pathway (Saade et al., 2017), raising the interesting idea that classical growth factors might contribute to centrosome maturation in dividing NPCs.

Centrosome asymmetry is also reflected in notable differences in the recruitment of signalling components. One of these determinants is the Mind bomb1 (Mib1) protein, which is essential for generating functional Notch ligands (Koo et al., 2005). Mib1 is enriched at the daughter centrosome during mitosis (Fig. 3A) and gets inherited by the prospective neuron in asymmetric divisions (Tozer et al., 2017). This asymmetry is determined through the association of Mib1 with centriolar satellites (Tozer et al., 2017). Asymmetric localization of Mib1 at the daughter centrosome is accompanied by an unexpected asymmetric enrichment of the satellite markers PCM1 and AZI1 (CEP131) at the daughter centrosome (Tozer et al., 2017). Disruption of this interaction leads to symmetric Mib1 localization in mitosis, reciprocal Notch activation between sister cells, and a reduction in asymmetric NPC divisions and neurogenesis. Interestingly, centriolar satellite proteins have also been shown to assemble with microcephaly-associated proteins and promote centriole duplication (Kodani et al., 2015).

Centrosome asymmetry also impacts on the capacity to reassemble a primary cilium; the daughter cell that inherits the mother centriole reassembles a cilium and responds to external stimuli, such as Shh and other growth factors, prior to its sister cell (Anderson and Stearns, 2009). In dividing NPCs, a portion of the ciliary membrane that is preferentially attached to the mother centriole is endocytosed at the onset of mitosis, persists through mitosis at one spindle pole (Fig. 3A), and is asymmetrically inherited by one daughter cell; this cells retains progenitor character (Paridaen et al., 2013; Saade et al., 2017; Wang et al., 2009). Hence, it appears that the presence of this ciliary membrane remnant speeds up primary cilium assembly and facilitates the integration of signals, which in turn helps to maintain asymmetric NPC division.
Centrosomes, and hence centrosome asymmetry, also determine the organization and final orientation of the mitotic spindle relative to the cell cortex during cell division (Negishi et al., 2016; Rebollo et al., 2007). As discussed above, mother and daughter centrosomes differ notably in the expansion of their PCM and in their MTOC activity at mitosis entry. As such, the mother centrosome organizes a microtubule aster that is larger than that of the daughter centrosome (Fig. 3A) (Negishi et al., 2016; Rebollo et al., 2007; Yamashita et al., 2007). Astral microtubules connect to the cell cortex via the NuMA/LGN/Gai protein complex, which, by recruiting motor proteins of the dynein/dynactin complex, pulls on astral microtubules; this, in turn, drives mitotic spindle movements and orientation (Konno et al., 2008; Lesage et al., 2010; Morin et al., 2007; Saadaoui et al., 2017). In dividing NPCs, mitotic spindle orientation is associated with the partitioning of apical membrane subdomains. At the luminal surface, membrane subdomains organize to form the apical junction complex where, among other proteins, Par3/6 and atypical protein kinase C (aPKC) localize (Kosodo et al., 2004; Marthiens and ffrench-Constant, 2009). By contrast, the junctional proteins $\mathrm{N}$-cadherin $(\mathrm{Cdh} 2), \alpha$-catenin and $\beta$-catenin are found in the sub-apical domain (Fig. 3A) (Kosodo et al., 2004; Marthiens and ffrench-Constant, 2009; Saade et al., 2017). During interphase, the apical junction complex drives the positioning of NPCs within the epithelium. However, when NPCs divide, the components of the apical junction complex redistribute depending on the orientation of the mitotic spindle and the fate of the daughter cells. As such, a symmetric distribution of apical membrane subdomains is associated with proliferative divisions in which both daughter cells remain within the VZ as NPCs. Minor changes in spindle orientation determine whether the cleavage plane bisects or bypasses the small apical domain of dividing NPCs (Fig. 3A) and hence determine the outcome of the division (Saade et al., 2017). Importantly, it has been shown that progenitors retaining the old mother centriole reorganize a new apical polarity complex and remain within the VZ (Das and Storey, 2014). By contrast, NPCs that inherit the daughter centrosome also inherit the old apical polarity complex, which becomes disorganized upon differentiation (Das and Storey, 2014; Kasioulis et al., 2017). Together, these findings suggest that asymmetric spindle orientation is associated with a reduction in symmetric divisions, premature cell cycle exit and premature neurogenesis, potentially leading to a microcephaly phenotype (Bultje et al., 2009; Lancaster and Knoblich, 2012; Shitamukai et al., 2011; Shitamukai and Matsuzaki, 2012; Wilcock et al., 2007).

Hence, all of the findings discussed above reinforce the idea that, from a centrosomal perspective, the default outcome of any cell division should be asymmetric. Overcoming these various centrosomal, and associated, asymmetries would be required to promote symmetric proliferative divisions and embryonic CNS growth, and failure to do so might lead to neurodevelopmental defects such as microcephaly. This is an important point to note, especially as much of the effort in this field has focused on the search for signals that instruct the switch to asymmetric division, which instead appears to be the default state for NPC division (and possibly for other dividing cells).

\section{New roles for classic growth factors in centrosome maturation during embryonic CNS growth}

In the growing CNS, the morphogenetic activity of secreted proteins that generate cell diversity (e.g. members of the Shh, Wnt and BMP families) is combined with their capacity to coordinate cell cycle progression by directly regulating discrete sets of genes that are key 
components of the cell cycle machinery (Alvarez-Medina et al., 2009; Cayuso et al., 2006; Molina and Pituello, 2017; Ulloa and Briscoe, 2007). In addition to such activities that ensure the maintenance of progenitor cell proliferation, these factors appear to modulate the mode of cell division adopted by NPCs and neurons.

Shh signalling, for example, has been shown to regulate the mode of motor neuron progenitor cell division within the developing spinal cord (Saade et al., 2013). By combining experimental data with mathematical modelling, it has been shown that the cell division mode switches sharply, from proliferative divisions to neurogenic divisions, with the sudden loss of Shh activity (Saade et al., 2013). In addition, maintaining Shh signalling artificially high is sufficient to prevent this developmental switch and to maintain symmetric proliferative divisions. This observation raised the question as to whether this Shh activity might impact on centrosome biology during NPC division. As introduced above, $\mathrm{Shh} /$ Gli activity in NPCs is sufficient to activate the expression of a cluster of centrosomal proteins, including centriolar and pericentriolar material and centrosome-associated proteins, that might contribute to centrosomal maturation and hence overcome intrinsic centrosome asymmetries (Saade et al., 2017). Among them, pericentrin, the expression of which is activated by Shh/Gli signalling, serves to dock an equal amount of protein kinase A (PKA) to both the mother and daughter centrosomes. PKA also exerts a downstream effect on processing of the Gli transcription factors so, at early developmental stages when Shh/Gli activity is high and proliferative divisions are predominant, the centrosomal localization of PKA becomes symmetric, leading to equal Shh activity in both daughter cells. As development proceeds, however, $\mathrm{Shh} /$ Gli activity decreases, pericentrin expression becomes low, and PKA remains associated with only the mother centrosome, leading to asymmetric Shh activity and neurogenic divisions (Fig. 3A). Disrupting the interaction of pericentrin with the centrosome leads to PKA mislocalization in mitosis and an increase in asymmetric neurogenic divisions (Saade et al., 2017). The expression of a number of additional centrosome proteins appears to be regulated by the Shh/Gli signalling pathway. These include CEP110 (CNTRL), which colocalizes with ninein and is involved in maturation of the daughter centrosome (Ou et al., 2002); ASPM, which concentrates at NPC mitotic spindle poles and is downregulated at the switch from symmetric proliferative to asymmetric neurogenic divisions (Fish et al., 2006); and PCM1, which is a component of centriolar satellites involved in the redistribution of molecular determinants (Tozer et al., 2017). Hence, centrosome maturation and the consequent regulation of the mode of NPC division should be added to the already long list of multiple roles played by Shh/Gli signalling during CNS development (Martí and Bovolenta, 2002).

The BMP/Smad and Wnt/ $\beta$-catenin signalling pathways also play major roles in regulating growth of the developing vertebrate nervous system (Le Dréau and Martí, 2012). Indeed, the mode of cell division adopted by interneurons in the developing spinal cord is dictated by different levels of activity of the canonical BMP effectors Smad1/5 (Le Dréau et al., 2014). Thus, analogous mechanisms regulating cell division, similar to those controlled by Shh, might be foreseen, particularly as signalling components of both pathways localize to centrosomes. Phosphorylated Smad1 ( $\mathrm{pSmad1}$ ), the effector of canonical BMP signalling, appears to be localized to centrosomes during cell division, although this pool of Smad1 protein (which is subjected to sequential phosphorylation by MAPK and glycogen synthase kinase 3) is targeted for degradation (Fuentealba et al., 2007). Moreover, pSmad proteins specifically targeted for proteasomal degradation are asymmetrically inherited preferentially by one daughter cell during cell division (Fig. 3B) (Fuentealba et al., 2008). Indeed, the proteasomal degradation of pSmad1 in the centrosome regulates the duration of the BMP signalling pathway, which in turn is known to maintain stem cell identity (Fuentealba et al., 2007; Le Dréau et al., 2014). This suggests that degradation mechanisms might be associated with the mother centrosome during asymmetric divisions (Fig. 3B).

Dividing NPCs in the mouse embryonic midbrain also show centrosomal localization of phosphorylated $\beta$-catenin - the effector of the canonical Wnt signalling pathway (Chilov et al., 2011) (Fig. 3B). Whether $\beta$-catenin is asymmetrically recruited to mitotic centrosomes in these cells, however, has not yet been addressed. Phosphorylated $\beta$-catenin also shows centrosomal localization in human embryonic stem cells (Fuentealba et al., 2008) and, in vitro, a localized Wnt signal can induce oriented cell divisions that generate distinct cell fates in embryonic stem cells (Habib et al., 2013). Moreover, in Caenorhabditis elegans, SYS-1/ $\beta$-catenin localizes to mitotic centrosomes in mother cells and is subjected to dynamic proteasome degradation (Vora and Phillips, 2015). In this context, the centrosomal localization negatively regulates SYS- $1 / \beta$-catenin levels and Wnt-dependent cell fate in daughter cells after division.

Hence, beyond age and structure, mother and daughter centrosomes appear to have different abilities to serve as hubs for the integration, duration and coordination of signalling pathways that are important for CNS growth.

\section{Centrosome dysfunction and microcephaly}

The consequences of centrosome dysfunction during development and how they contribute to human diseases are highlighted by the study of autosomal recessive primary microcephaly (MCPH). $\mathrm{MCPH}$ is a genetically heterogeneous neurodevelopmental disorder characterized by a small CNS at birth and nonprogressive intellectual disability. Many of the causative genes for the 20 loci mapped to date (MCPH1-MCPH20) in various populations around the globe encode centriole/centrosome or kinetochore/spindle pole proteins that are involved in centriole biogenesis, centrosome maturation, cytokinesis, centromere and kinetochore function (Table 1). This indicates that centrosome dysfunction is one of the main causes of MCPH (Jayaraman et al., 2018; Nano and Basto, 2017). Moreover, additional microcephaly phenotypes are associated with centrosome proteins, including CEP63, PCNT, NIN, POC1A (Table 1), establishing a strong genetic link between centrosome dysfunction during development and the aetiology of microcephaly. What remains to be resolved, however, is why brain size in particular is so vulnerable to centrosome mutations; centrosome dysfunction found in $\mathrm{MCPH}$ mostly leads to architecturally normal but smaller brains, in most cases without affecting body size. It thus appears that, compared with other organs, size regulation in the CNS might rely more on the tightly controlled mode of cell divisions that occur during developmental stages.

\section{Conclusions}

As we have reviewed here, recent research in animal models has started to reveal the multiple roles played by centrosomes during embryonic CNS growth and neurogenesis. Centrosomes are confined to the apical pole of NPCs where they serve as a basal body for the primary cilium. As such, they regulate the exposure of cells to the growth factor signalling-rich microenvironment of the NT lumen. The subsequent integration of growth factor signals during the G1 phase of the cell cycle results in the regulated 
Table 1. The MCPH1-20 loci, their gene products and protein functions

\begin{tabular}{|c|c|c|c|c|}
\hline Locus & $\begin{array}{l}\text { Gene } \\
\text { product }\end{array}$ & Alternative names and symbols & Protein function and localization & OMIM \\
\hline MCPH1 & $\mathrm{MCPH} 1$ & $\begin{array}{l}\text { Microcephalin; BRCT-repeat inhibitor of TERT } \\
\text { expression 1; BRIT1 }\end{array}$ & Regulates chromosome condensation & 607117 \\
\hline MCPH3 & CEP215 & $\begin{array}{l}\text { Centrosomal protein, } 215-\mathrm{kD} \text {; CDK5 regulatory } \\
\text { subunit-associated protein; CDK5RAP2 }\end{array}$ & $\begin{array}{l}\text { Localizes to the centrosome and to the spindle poles } \\
\text { during mitosis }\end{array}$ & 608201 \\
\hline $\mathrm{MCPH} 4$ & CASC5 & Kinetochore scaffold $1 ; \mathrm{KNL} 1$ & Localizes to the kinetochore & 609173 \\
\hline MCPH5 & ASPM & Abnormal spindle-like, microcephaly-associated & Essential for mitotic spindle assembly/function & 605481 \\
\hline $\mathrm{MCPH} 7$ & STIL & SCL/TAL1-interrupting locus & Localizes to PCM, regulates centriole duplication & 181590 \\
\hline MCPH8 & CEP135 & Centrosomal protein, $135-\mathrm{kD}$ & $\begin{array}{l}\text { Forms the core centriole structure, regulates early } \\
\text { centriole assembly }\end{array}$ & 611423 \\
\hline МСРH9 & CEP152 & Centrosomal protein, $152-\mathrm{kD}$ & Core protein of the centrosome & 613529 \\
\hline MCPH10 & ZNF335 & Zinc finger protein 335; NRC-interacting factor; NIF1 & $\begin{array}{l}\text { Component of the trithorax H3K4-methylation chromatin } \\
\text { remodelling complex }\end{array}$ & 610827 \\
\hline MCPH13 & CENPE & Centromeric protein $\mathrm{E}$ & Kinetochore-associated kinesin-like motor protein & 117143 \\
\hline MCPH14 & SASS6 & SAS- 6 centriolar assembly protein & Functions in procentriole formation & 609321 \\
\hline MCPH15 & MFSD2A & Major facilitator superfamily domain-containing protein $2 \mathrm{~A}$ & Sodium-dependent lysophosphatidylcholine transporter & 614397 \\
\hline MCPH16 & ANKLE2 & $\begin{array}{l}\text { Ankyrin repeat- and LEM domain-containing protein } 2 \text {; } \\
\text { LEM domain-containing protein } 4 \text {; LEM4 }\end{array}$ & $\begin{array}{l}\text { Regulates reassembly of the nuclear envelope at } \\
\text { anaphase }\end{array}$ & 616062 \\
\hline $\mathrm{MCPH} 17$ & CIT & $\begin{array}{l}\text { Citron } \mathrm{RHO} \text {-interacting serine/threonine kinase; } \\
\text { serine/threonine protein kinase } 21 ; \text { STK21 }\end{array}$ & Essential for cytokinesis & 605629 \\
\hline MCPH18 & WDFY3 & WD repeat- and FYVE domain-containing protein 3 & $\begin{array}{l}\text { Organizes misfolded ubiquitylated proteins into bodies to } \\
\text { be degraded by autophagy }\end{array}$ & 617485 \\
\hline MCPH19 & COPB2 & Coatomer protein complex, subunit $\beta 2$ & $\begin{array}{l}\text { Subunit of the Golgi coatomer complex, necessary for } \\
\text { retrograde intracellular trafficking }\end{array}$ & 606990 \\
\hline $\mathrm{MCPH} 20$ & KIF14 & Kinesin family member 14 & $\begin{array}{l}\text { Microtubule-associated motor, plays an important role in } \\
\text { cell division }\end{array}$ & 611279 \\
\hline
\end{tabular}

OMIM, Online Mendelian Inheritance in Man

Loci beneath the line relate to other centrosome proteins with associated microcephaly phenotypes.

expression of multiple targets including genes involved in centrosome maturation. Hence, by controlling symmetric centrosomal protein assembly, growth factors can overcome the intrinsic asymmetry of the centrosome during NPC division, thereby promoting self-expanding symmetric divisions and CNS growth. Importantly, the failure to overcome such intrinsic cell division asymmetries, and thus the failure to ensure appropriate cell divisions during early CNS growth, may be responsible for neurodevelopmental disorders such as primary microcephaly.

Moving forward, we propose that we should turn our attention to the search for instructive signals that can overcome these intrinsic asymmetries in NPC divisions. As we have highlighted here, classical growth factors might be key players. For example, a role for Shh has recently been revealed and requires further investigation. It will also be important to understand how molecular components of the Wnt and BMP signalling pathways are integrated into the mitotic centrosome and whether they affect NPC modes of division. It is likely that additional regulatory mechanisms that remain to be discovered are also involved, and their characterization might expand our knowledge of how, from a centrosomal perspective, classical growth factors contribute to defining the division mode of NPCs. Do such components participate directly in the intrinsic functions of the centrosome? Does the centrosome serve as a hub for the integration, duration and distribution of these signals in NPCs after division? These key open questions need to be answered in order to fully understand CNS growth from a centrosomal point of view.

\section{Acknowledgements}

We apologize to colleagues whose work is not included owing to space constraints We thank the E.M. laboratory members for useful discussions.

\section{Competing interests}

The authors declare no competing or financial interests.

\section{Funding}

The work in E.M.'s laboratory is supported by grants from Ministerio de Economía, Industria y Competitividad, Gobierno de España (BFU2016-77498-P and BFU2016-81887-REDT). E.G.-G. holds a Predoctoral Scholarship BES-2014068589, J.B.-A. holds a Predoctoral Scholarship BES-2017-080050 from Ministerio de Economía, Industria y Competitividad, Gobierno de España.

\section{References}

Addison, M. and Wilkinson, D. G. (2016). Segment identity and cell segregation in the vertebrate hindbrain. Curr. Top. Dev. Biol. 117, 581-596. 
Almada, E., Tonucci, F. M., Hidalgo, F., Ferretti, A., Ibarra, S., Pariani, A., Vena, R., Favre, C., Girardini, J., Kierbel, A. et al. (2017). Akap350 recruits Eb1 to the spindle poles, ensuring proper spindle orientation and lumen formation in 3D epithelial cell cultures. Sci. Rep. 7, 14894

Alvarez-Medina, R., Le Dreau, G., Ros, M. and Marti, E. (2009). Hedgehog activation is required upstream of Wnt signalling to control neural progenitor proliferation. Development 136, 3301-3309.

Anderson, C. T. and Stearns, T. (2009). Centriole age underlies asynchronous primary cilium growth in mammalian cells. Curr. Biol. 19, 1498-1502

Buchman, J. J., Tseng, H.-C., Zhou, Y., Frank, C. L., Xie, Z. and Tsai, L.-H. (2010). Cdk5rap2 interacts with pericentrin to maintain the neural progenitor poo in the developing neocortex. Neuron 66, 386-402.

Bultje, R. S., Castaneda-Castellanos, D. R., Jan, L. Y., Jan, Y.-N., Kriegstein, A. R. and Shi, S.-H. (2009). Mammalian Par3 regulates progenitor cell asymmetric division via notch signaling in the developing neocortex. Neuron 63, 189-202.

Cayuso, J., Ulloa, F., Cox, B., Briscoe, J. and Marti, E. (2006). The Sonic hedgehog pathway independently controls the patterning, proliferation and survival of neuroepithelial cells by regulating Gli activity. Development 133 517-528

Chilov, D., Sinjushina, N., Rita, H., Taketo, M. M., Mäkelä, T. P. and Partanen, J. (2011). Phosphorylated beta-catenin localizes to centrosomes of neuronal progenitors and is required for cell polarity and neurogenesis in developing midbrain. Dev. Biol. 357, 259-268.

Cohen, M., Briscoe, J. and Blassberg, R. (2013). Morphogen interpretation: the transcriptional logic of neural tube patterning. Curr. Opin. Genet. Dev. 23, 423-428.

Dammermann, A. and Merdes, A. (2002). Assembly of centrosomal proteins and microtubule organization depends on PCM-1. J. Cell Biol. 159, 255-266.

Das, R. M. and Storey, K. G. (2014). Apical abscission alters cell polarity and dismantles the primary cilium during neurogenesis. Science 343, 200-204

Del Bene, F., Wehman, A. M., Link, B. A. and Baier, H. (2008). Regulation of neurogenesis by interkinetic nuclear migration through an apical-basal notch gradient. Cell 134, 1055-1065.

Dubreuil, V., Marzesco, A.-M., Corbeil, D., Huttner, W. B. and WilschBräuninger, M. (2007). Midbody and primary cilium of neural progenitors release extracellular membrane particles enriched in the stem cell marker prominin-1. J. Cell Biol. 176, 483-495.

Feng, Y., Olson, E. C., Stukenberg, P. T., Flanagan, L. A., Kirschner, M. W. and Walsh, C. A. (2000). LIS1 regulates CNS lamination by interacting with mNudE, a central component of the centrosome. Neuron 28, 665-679.

Fish, J. L., Kosodo, Y., Enard, W., Paabo, S. and Huttner, W. B. (2006). Aspm specifically maintains symmetric proliferative divisions of neuroepithelial cells Proc. Natl. Acad. Sci. USA 103, 10438-10443.

Florio, M., Borrell, V. and Huttner, W. B. (2017). Human-specific genomic signatures of neocortical expansion. Curr. Opin. Neurobiol. 42, 33-44.

Fuentealba, L. C., Eivers, E., Ikeda, A., Hurtado, C., Kuroda, H., Pera, E. M. and De Robertis, E. M. (2007). Integrating patterning signals: Wnt/GSK3 regulates the duration of the BMP/Smad1 signal. Cell 131, 980-993.

Fuentealba, L. C., Eivers, E., Geissert, D., Taelman, V. and De Robertis, E. M. (2008). Asymmetric mitosis: unequal segregation of proteins destined for degradation. Proc. Natl. Acad. Sci. USA 105, 7732-7737.

Gai, M., Bianchi, F. T., Vagnoni, C., Vernì, F., Bonaccorsi, S., Pasquero, S., Berto, G. E., Sgrò, F., Chiotto, A. M. A., Annaratone, L. et al. (2016). ASPM and CITK regulate spindle orientation by affecting the dynamics of astral microtubules. EMBO Rep. 17, 1396-1409.

Ge, X., Frank, C. L., Calderon de Anda, F. and Tsai, L.-H. (2010). Hook3 interacts with PCM1 to regulate pericentriolar material assembly and the timing of neurogenesis. Neuron 65, 191-203.

Gillingham, A. K. and Munro, S. (2000). The PACT domain, a conserved centrosomal targeting motif in the coiled-coil proteins AKAP450 and pericentrin. EMBO Rep. 1, 524-529.

Gilmore, E. C. and Walsh, C. A. (2013). Genetic causes of microcephaly and lessons for neuronal development. Wiley Interdiscip. Rev. Dev. Biol. 2, 461-478.

Goetz, S. C. and Anderson, K. V. (2010). The primary cilium: a signalling centre during vertebrate development. Nat. Rev. Genet. 11, 331-344.

Graser, S., Stierhof, Y.-D., Lavoie, S. B., Gassner, O. S., Lamla, S., Le Clech, M. and Nigg, E. A. (2007). Cep164, a novel centriole appendage protein required for primary cilium formation. J. Cell Biol. 179, 321-330.

Greene, N. D. E. and Copp, A. J. (2014). Neural tube defects. Annu. Rev. Neurosci. $37,221-242$

Gupta, S. and Sen, J. (2016). Roof plate mediated morphogenesis of the forebrain new players join the game. Dev. Biol. 413, 145-152

Habib, S. J., Chen, B.-C., Tsai, F.-C., Anastassiadis, K., Meyer, T., Betzig, E. and Nusse, R. (2013). A localized Wnt signal orients asymmetric stem cell division in vitro. Science 339, 1445-1448.

Hatakeyama, J., Wakamatsu, Y., Nagafuchi, A., Kageyama, R., Shigemoto, R. and Shimamura, K. (2014). Cadherin-based adhesions in the apical endfoot are required for active Notch signaling to control neurogenesis in vertebrates. Development 141, 1671-1682.
Heide, M., Long, K. R. and Huttner, W. B. (2017). Novel gene function and regulation in neocortex expansion. Curr. Opin. Cell Biol. 49, 22-30.

Hirota, T., Kunitoku, N., Sasayama, T., Marumoto, T., Zhang, D., Nitta, M., Hatakeyama, K. and Saya, H. (2003). Aurora-A and an interacting activator, the LIM protein Ajuba, are required for mitotic commitment in human cells. Cell 114, 585-598.

Hoyer-Fender, S. (2010). Centriole maturation and transformation to basal body. Semin. Cell Dev. Biol. 21, 142-147.

Insolera, R., Bazzi, H., Shao, W., Anderson, K. V. and Shi, S. H. (2014). Cortical neurogenesis in the absence of centrioles. Nat. Neurosci. 17, 1528-1535.

Ishikawa, H., Kubo, A., Tsukita, S. and Tsukita, S. (2005). Odf2-deficient mothe centrioles lack distal/subdistal appendages and the ability to generate primary cilia. Nat. Cell Biol. 7, 517-524.

Jackman, M., Lindon, C., Nigg, E. A. and Pines, J. (2003). Active cyclin B1-Cdk1 first appears on centrosomes in prophase. Nat. Cell Biol. 5, 143-148.

Jayaraman, D., Kodani, A., Gonzalez, D. M., Mancias, J. D., Mochida, G. H. Vagnoni, C., Johnson, J., Krogan, N., Harper, J. W., Reiter, J. F. et al. (2016). Microcephaly proteins Wdr62 and Aspm define a mother centriole complex regulating centriole biogenesis, apical complex, and cell fate. Neuron 92 , 813-828.

Jayaraman, D., Bae, B.-I. and Walsh, C. A. (2018). The genetics of primary microcephaly. Annu. Rev. Genomics Hum. Genet. 19, 177-200.

Johnson, M. B. and Walsh, C. A. (2017). Cerebral cortical neuron diversity and development at single-cell resolution. Curr. Opin. Neurobiol. 42, 9-16.

Kasioulis, I., Das, R. M. and Storey, K. G. (2017). Inter-dependent apical microtubule and actin dynamics orchestrate centrosome retention and neuronal delamination. eLife 6, e26215.

Kodani, A., Yu, T. W., Johnson, J. R., Jayaraman, D., Johnson, T. L., Al-Gazali, L., Sztriha, L., Partlow, J. N., Kim, H., Krup, A. L. et al. (2015). Centriolar satellites assemble centrosomal microcephaly proteins to recruit CDK2 and promote centriole duplication. eLife 4 .

Konno, D., Shioi, G., Shitamukai, A., Mori, A., Kiyonari, H., Miyata, T. and Matsuzaki, F. (2008). Neuroepithelial progenitors undergo LGN-dependent planar divisions to maintain self-renewability during mammalian neurogenesis. Nat. Cell Biol. 10, 93-101.

Koo, B.-K., Lim, H. S., Song, R., Yoon, M. J., Yoon, K. J., Moon, J. S., Kim, Y. W. Kwon, M. C., Yoo, K. W., Kong, M. P. et al. (2005). Mind bomb 1 is essential for generating functional Notch ligands to activate Notch. Development 132 3459-3470.

Kosodo, Y., Röper, K., Haubensak, W., Marzesco, A.-M., Corbeil, D. and Huttner, W. B. (2004). Asymmetric distribution of the apical plasma membrane during neurogenic divisions of mammalian neuroepithelial cells. EMBO J.23, 2314-2324.

Kosodo, Y., Suetsugu, T., Suda, M., Mimori-Kiyosue, Y., Toida, K., Baba, S. A., Kimura, A. and Matsuzaki, F. (2011). Regulation of interkinetic nuclear migration by cell cycle-coupled active and passive mechanisms in the developing brain. EMBO J. 30, 1690-1704.

Lancaster, M. A. and Knoblich, J. A. (2012). Spindle orientation in mammalian cerebral cortical development. Curr. Opin. Neurobiol. 22, 737-746.

Langman, J., Guerrant, R. L. and Freeman, B. G. (1966). Behavior of neuroepithelial cells during closure of the neural tube. J. Comp. Neurol. 127, 399-411. Le Dréau, G. and Martí, E. (2012). Dorsal-ventral patterning of the neural tube: a tale of three signals. Dev. Neurobiol. 72, 1471-1481.

Le Dréau, G., Saade, M., Gutiérrez-Vallejo, I. and Martí, E. (2014). The strength of SMAD1/5 activity determines the mode of stem cell division in the developing spinal cord. J. Cell Biol. 204, 591-605.

Lee, H. O. and Norden, C. (2013). Mechanisms controlling arrangements and movements of nuclei in pseudostratified epithelia. Trends Cell Biol. 23, 141-150.

Lesage, B., Gutierrez, I., Martí, E. and Gonzalez, C. (2010). Neural stem cells: the need for a proper orientation. Curr. Opin. Genet. Dev. 20, 438-442.

Mahen, R. and Venkitaraman, A. R. (2012). Pattern formation in centrosome assembly. Curr. Opin. Cell Biol. 24, 14-23.

Marthiens, V. and ffrench-Constant, C. (2009). Adherens junction domains are split by asymmetric division of embryonic neural stem cells. EMBO Rep. 10 515-520.

Martí, E. and Bovolenta, P. (2002). Sonic hedgehog in CNS development: one signal, multiple outputs. Trends Neurosci. 25, 89-96.

Molina, A. and Pituello, F. (2017). Playing with the cell cycle to build the spinal cord Dev. Biol. 432, 14-23.

Morin, X., Jaouen, F. and Durbec, P. (2007). Control of planar divisions by the G-protein regulator LGN maintains progenitors in the chick neuroepithelium. Nat. Neurosci. 10, 1440-1448.

Nano, M. and Basto, R. (2017). Consequences of centrosome dysfunction during brain development. Adv. Exp. Med. Biol. 1002, 19-45.

Negishi, T., Miyazaki, N., Murata, K., Yasuo, H. and Ueno, N. (2016). Physical association between a novel plasma-membrane structure and centrosome orients cell division. eLife 5, e16550.

Ohata, S., Aoki, R., Kinoshita, S., Yamaguchi, M., Tsuruoka-Kinoshita, S. Tanaka, H., Wada, H., Watabe, S., Tsuboi, T., Masai, I. et al. (2011). Dual roles 
of Notch in regulation of apically restricted mitosis and apicobasal polarity of neuroepithelial cells. Neuron 69, 215-230.

Ou, Y. Y., Mack, G. J., Zhang, M. and Rattner, J. B. (2002). CEP110 and ninein are located in a specific domain of the centrosome associated with centrosome maturation. J. Cell Sci. 115, 1825-1835.

Paridaen, J. T. M. L., Wilsch-Bräuninger, M. and Huttner, W. B. (2013). Asymmetric inheritance of centrosome-associated primary cilium membrane directs ciliogenesis after cell division. Cell 155, 333-344

Rebollo, E., Sampaio, P., Januschke, J., Llamazares, S., Varmark, H. and González, C. (2007). Functionally unequal centrosomes drive spindle orientation in asymmetrically dividing Drosophila neural stem cells. Dev. Cell 12, 467-474.

Reinsch, S. and Gonczy, P. (1998). Mechanisms of nuclear positioning. J. Cell Sci. 111, 2283-2295.

Saadaoui, M., Konno, D., Loulier, K., Goiame, R., Jadhav, V., Mapelli, M. Matsuzaki, F. and Morin, X. (2017). Loss of the canonical spindle orientation function in the Pins/LGN homolog AGS3. EMBO Rep. 18, 1509-1520.

Saade, M., Gutiérrez-Vallejo, I., Le Dréau, G., Rabadán, M. A., Miguez, D. G., Buceta, J. and Martí, E. (2013). Sonic hedgehog signaling switches the mode of division in the developing nervous system. Cell Rep. 4, 492-503.

Saade, M., Gonzalez-Gobartt, E., Escalona, R., Usieto, S. and Martí, E. (2017). Shh-mediated centrosomal recruitment of PKA promotes symmetric proliferative neuroepithelial cell division. Nat. Cell Biol. 19, 493-503.

Sauer, F. C. (1935). Mitosis in the neural tube. J. Comp. Neurol. 62, 377-405

Schenk, J., Wilsch-Brauninger, M., Calegari, F. and Huttner, W. B. (2009). Myosin II is required for interkinetic nuclear migration of neural progenitors. Proc. Natl. Acad. Sci. USA 106, 16487-16492.

Schmidt, K. N., Kuhns, S., Neuner, A., Hub, B., Zentgraf, H. and Pereira, G. (2012). Cep164 mediates vesicular docking to the mother centriole during early steps of ciliogenesis. J. Cell Biol. 199, 1083-1101.

Shitamukai, A. and Matsuzaki, F. (2012). Control of asymmetric cell division of mammalian neural progenitors. Dev. Growth Differ. 54, 277-286.

Shitamukai, A., Konno, D. and Matsuzaki, F. (2011). Oblique radial glial divisions in the developing mouse neocortex induce self-renewing progenitors outside the germinal zone that resemble primate outer subventricular zone progenitors. J. Neurosci. 31, 3683-3695.

Sousa, V. H. and Fishell, G. (2010). Sonic hedgehog functions through dynamic changes in temporal competence in the developing forebrain. Curr. Opin. Genet Dev. 20, 391-399.

Strzyz, P. J., Lee, H. O., Sidhaye, J., Weber, I. P., Leung, L. C. and Norden, C. (2015). Interkinetic nuclear migration is centrosome independent and ensures apical cell division to maintain tissue integrity. Dev. Cell 32, 203-219.

Tanaka, T., Serneo, F. F., Higgins, C., Gambello, M. J., Wynshaw-Boris, A. and Gleeson, J. G. (2004). Lis1 and doublecortin function with dynein to mediate coupling of the nucleus to the centrosome in neuronal migration. J. Cell Biol. 165 709-721.

Tozer, S., Baek, C., Fischer, E., Goiame, R. and Morin, X. (2017). Differential routing of mindbomb1 via centriolar satellites regulates asymmetric divisions of neural progenitors. Neuron 93, 542-551.e544.

Tsai, J.-W., Lian, W.-N., Kemal, S., Kriegstein, A. R. and Vallee, R. B. (2010). Kinesin 3 and cytoplasmic dynein mediate interkinetic nuclear migration in neural stem cells. Nat. Neurosci. 13, 1463-1471.

Tsuda, S., Kitagawa, T., Takashima, S., Asakawa, S., Shimizu, N., Mitani, H., Shima, A., Tsutsumi, M., Hori, H., Naruse, K. et al. (2010). FAK-mediated extracellular signals are essential for interkinetic nuclear migration and planar divisions in the neuroepithelium. J. Cell Sci. 123, 484-496.

Ulloa, F. and Briscoe, J. (2007). Morphogens and the control of cell proliferation and patterning in the spinal cord. Cell Cycle 6, 2640-2649.

Ulloa, F. and Marti, E. (2010). Wnt won the war: antagonistic role of Wnt over Shh controls dorso-ventral patterning of the vertebrate neural tube. Dev. Dyn 239, 69-76.

Vora, S. and Phillips, B. T. (2015). Centrosome-associated degradation limits beta-catenin inheritance by daughter cells after asymmetric division. Curr. Biol. 25, 1005-1016.

Wang, X., Tsai, J.-W., Imai, J. H., Lian, W.-N., Vallee, R. B. and Shi, S.-H. (2009). Asymmetric centrosome inheritance maintains neural progenitors in the neocortex. Nature 461, 947-955.

Welburn, J. P. I. and Cheeseman, I. M. (2012). The microtubule-binding protein Cep170 promotes the targeting of the kinesin-13 depolymerase Kif2b to the mitotic spindle. Mol. Biol. Cell 23, 4786-4795.

Wilcock, A. C., Swedlow, J. R. and Storey, K. G. (2007). Mitotic spindle orientation distinguishes stem cell and terminal modes of neuron production in the early spinal cord. Development 134, 1943-1954

Wilsch-Bräuninger, M., Florio, M. and Huttner, W. B. (2016). Neocortex expansion in development and evolution - from cell biology to single genes. Curr. Opin. Neurobiol. 39, 122-132.

Xie, Z., Moy, L. Y., Sanada, K., Zhou, Y., Buchman, J. J. and Tsai, L.-H. (2007) Cep120 and TACCs control interkinetic nuclear migration and the neural progenitor pool. Neuron 56, 79-93.

Yamashita, Y. M., Mahowald, A. P., Perlin, J. R. and Fuller, M. T. (2007) Asymmetric inheritance of mother versus daughter centrosome in stem cell division. Science 315, 518-521.

Zhang, X., Lei, K., Yuan, X., Wu, X., Zhuang, Y., Xu, T., Xu, R. and Han, M. (2009). SUN1/2 and Syne/Nesprin-1/2 complexes connect centrosome to the nucleus during neurogenesis and neuronal migration in mice. Neuron 64, 173-187. 